\title{
Délibérations du Comité central de la FMH
}

St. Lors de sa séance du 2 mai 2000, après une discussion en conclave sur le TarMed, le Comité central (CC) a traité entre autres les affaires suivantes:

\section{Réforme des structures}

Bureau d'expertises extrajudiciaires de la FMH: désignation d'experts par le CC de la FMH

Un médecin impliqué dans un cas d'expertise a récusé les deux experts désignés par le médiateur de l'Union suisse des sociétés chirurgicales. Or, arguant que les soupçons de ce médecin à l'endroit des experts étaient infondés, le médiateur les a maintenus. Le règlement du Bureau d'expertises prévoit qu'en tel cas, il appartient au CC de trancher. Celui-ci confirme la nomination des deux experts proposés.

\section{Formation postgraduée et continue}

1. Concept de certification des établissements de formation postgraduée

On compte actuellement parmi les hôpitaux, instituts et cabinets médicaux de Suisse quelque 1400 établissements de formation reconnus par la FMH pour la formation en vue d'un titre de spécialiste. Or, l'acceptation des accords bilatéraux entraînant la révision de la loi sur l'exercice des professions médicales et celle de la Réglementation pour la formation postgraduée imposent une nouvelle méthode d'évaluation, celle en vigueur jusqu'ici, basée sur la déclaration personnelle, ne répondant plus aux nouvelles exigences. En 1997 déjà, le CC avait opté pour un procédé d'évaluation à trois échelons. Un essai pilote a eu lieu depuis lors dans dix établissements de formation.

Le CC décide de mettre sur pied un groupe de travail chargé de la création d'un office de certification des établissements de formation postgraduée et de l'établissement d'une liste des priorités.
2. Création d'un groupe de travail pour la RFC

L'adoption de la Réglementation pour la formation continue (RFC) par la Chambre médicale a obligé les sociétés de discipline médicales à établir un programme de formation continue. Toutes l'ont fait, mais ceux-ci varient considérablement en qualité et en quantité d'une discipline à l'autre. Un projet prévoit la mise sur pied d'un groupe de travail chargé du réexamen et de la révision de la RFC. Sa tâche consisterait également à revoir et à harmoniser les programmes de formation continue pour les titres de spécialiste, les formations approfondies, les attestations de formation complémentaire et les certificats d'aptitude technique. Les critères approuvés par la Chambre médicale pour les programmes de formation continue devront à leur tour être réexaminés et adaptés aux nouvelles exigences.

Le CC opte pour la mise sur pied d'un tel groupe qui sera chargé des mandats susmentionnés.

\section{Révision des titres de spécialiste}

Sous réserve de quelques retouches rédactionnelles, le CC approuve les programmes de formation en chirurgie pédiatrique, en psychiatrie d'enfants et d'adolescents et en neurochirurgie et décide de leur mise en vigueur au $1^{\mathrm{er}}$ juillet 2000.

\section{Certificat d'aptitude technique en thérapie} neurale

Le CC prend acte de la lettre du Département de la formation postgraduée et continue de la FMH informant la Société médicale suisse de thérapie neurale selon Huneke (SMSTN) et la Société médicale suisse de médecine régulatrice - Thérapie neurale (SRN) que le programme de formation en thérapie neurale approuvé l'année dernière par la Chambre médicale ne sera pas modifié pour le moment. Le CC se déclare prêt toutefois à étendre la notion de thérapie neurale dans le contexte de l'Ordonnance sur les prestations de l'assurance des soins (OPAS) si la Commission fédérale des prestations (CFP) devait adopter le programme de la SRN et exiger une modification de l'ordonnance en conséquence.

Deutsch erschienen in Nr. 24/2000 